\title{
An Experimental Investigation of Presupposition Projection in Conditional Sentences*
}

\author{
Jacopo Romoli \\ Harvard University
}

\author{
Yasutada Sudo \\ Massachusetts Institute of Technology
}

\author{
Jesse Snedeker \\ Harvard University
}

\begin{abstract}
Conditional sentences with a presupposition trigger in the consequentif $\phi$, then $\psi_{p}$ - show peculiar projection properties. That is, the entire sentence suggests that $p$ in some cases and that $\phi \rightarrow p$ in other cases. We report on the results of two sentence-picture matching experiments that indicate that the perceived dependence of the presupposition $p$ on the antecedent $\phi$ affects which inference is made. The results are also used to compare two major theories of presupposition projection, and we argue that the theory that postulates $\phi \rightarrow p$ as the presupposition of all conditional sentences explains the results more straightforwardly than its competitor.
\end{abstract}

Keywords: Presuppositions, Presupposition projection, Proviso problem, Conditionals

\section{Introduction}

Presuppositions are a type of inference associated with sentences containing certain words or grammatical constructions (for overviews see Chierchia \& McConnellGinet 2000; Kadmon 2001; Beaver \& Geurts 2011). For example, a simple positive sentence with a possessive NP necessarily presupposes that the possessor owns the possessee. More concretely, (1) presupposes that Paul owns a Bible.

(1) Paul will read his Bible tonight.

When a presupposition $p$ stems from a word or construction $\alpha$, we say that $\alpha$ "triggers" the presupposition $p$. It is known that the class of presupposition triggers is

* We are indebted to the following people for valuable discussions, critical comments and helpful suggestions at various stages of the project reported in this paper: David Beaver, Alejandro PérezCarballo, Greg Carlson, Ivano Caponigro, Emmanuel Chemla, Gennaro Chierchia, Tim O’Donnell, Danny Fox, Michael Franke, Ben George, Josh Hartshorne, Larry Horn, Manizeh Khan, Dan Lassiter, Clemens Mayr, Hazel Pearson, Philippe Schlenker, Greg Scontras and Raj Singh. We also thank the three SALT reviewers, and the three ESSLLI 2011 Student Session reviewers as well as the audiences of SALT 21, ESSLLI 2011 Student Session, and the Language and Cognition Group at Harvard. 
diverse and heterogeneous in certain respects. In order to avoid possible complications regarding the variation among presupposition triggers, we will exclusively look at presuppositions triggered by possessive NPs in this paper.

The main interest of the present paper is the so-called projection problem of presuppositions (Beaver 2001; Geurts 1999; George 2008; Heim 1983, 1992; Singh 2008; Schlenker 2008, 2009 among others), which is the problem of constructing a general procedure that predicts the presuppositions of an arbitrary sentence based upon the presuppositions of its parts and the way they are combined. In the present paper, we will focus on the presuppositions of conditional sentences with a presupposition trigger in the consequent, schematically represented as if $\phi$, then $\psi_{p}$, where $p$ is the presupposition triggered in $\psi$.

The projection properties of conditional sentences have been at the center of the debate in the literature (Geurts 1999; Beaver 2001; von Fintel 2008; Pérez-Carballo 2009; Singh 2008; Schlenker 2010). The problem posed by conditional sentences is now widely known as the proviso problem (Geurts 1999). Simply put, conditional sentences of the form if $\phi$, then $\psi_{p}$ give rise to two different kinds of inferences that are based on the presupposition $p$. That is, in some cases they seem to simply presuppose $p$, while in other cases they seem to presuppose a weaker proposition $\phi \rightarrow p$. The following examples taken from Pérez-Carballo 2009 illustrate this.

(2) If Paul is not tired, then he will read his Bible tonight.

a. If Paul is not tired, then he has a Bible.

b. Paul has a Bible.

(3) If Paul is a devout Catholic, then he will read his Bible tonight.

a. If Paul is a devout Catholic, then he has a Bible.

b. Paul has a Bible.

Intuitively, (2) seems to presuppose (2b) rather than (2a), while (3) seems to presuppose (3a) and typically not (3b). As the only difference between the two examples is the antecedent clause, the antecedent clause must play an important role in the present phenomenon.

A terminological remark is in order here. According to some theories, one of the two inferences is not analyzed as a true presupposition but as an inference of a different sort. To remain theoretically neutral, we will call inferences like (2b) "non-conditional inferences" and those like (3a) "conditional inferences".

It is important to notice at this moment that as Singh $(2007,2008)$ and Schlenker (2011b) point out, the proviso problem can be decomposed into two subproblems that can be tackled separately. More specifically, any complete theory of presupposition projection should be equipped with mechanisms to generate both conditional and non-conditional inferences, and also it should be able to predict which inference 
Romoli, Sudo and Snedeker

obtains when. Here we call these subproblems the Generation Problem and the Selection Problem. ${ }^{1}$

(4) a. Generation Problem: How are the conditional and non-conditional inferences generated in if $\phi$, then $\psi_{p}$ ?

b. Selection Problem: How is the inference that actually obtains selected? ${ }^{2}$

In this paper we present the results of two picture selection experiments which aim at answering the Selection Problem. In particular, we show that the dependency of the presupposition $p$ on the antecedent clause $\phi$ plays a key role in predicting which inference obtains. In addition we also claim that the experimental results favor the theory of presupposition projection that postulates $\phi \rightarrow p$ as a presupposition of if $\phi$, then $\psi_{p}$ over the theory that analyzes the conditional inference as a mere entailment. The organization of the paper is as follows: in Section 2, we introduce two theories of presupposition projection that generate conditional inferences as presuppositions and as mere entailments. In Section 3, we report on Experiment 1 and in Section 4 on Experiment 2. The results of these two experiments are compared in Section 5. In Section 6, we summarize and discuss further directions.

\section{Two Theories of Conditional Inference}

In this paper, we focus on two major theories of presupposition projection in conditional sentences. According to one theory, the conditional inference is a presupposition for all conditional sentences, while according to the other theory, the conditional inference arises in special cases as an entailment. Each of these theories has several distinct incarnations with non-trivial differences, but we will abstract away from those aspects that are orthogonal to the question at hand.

\subsection{Conditional Presupposition Theory}

One theory, which we call the conditional presupposition theory, assigns a conditional presupposition $\phi \rightarrow p$ to all conditional sentences of the form if $\phi$, then $\psi_{p}$ (Beaver 2001; Karttunen 1974; Heim 1983; Schlenker 2008, 2009, 2011a; Singh 2007, 2008). Whether this is the only possible presupposition or not depends on the different versions of this theory, but in all versions the conditional inference $\phi \rightarrow p$ is straightforwardly accounted for. For the non-conditional inference $p$, there

1 Schlenker (2011b) calls the former problem the 'strengthening problem'. We use the more neutral term here.

2 By 'selection', we do not mean to imply that the two inferences are both available in every case. In fact, some of the theories described below lend themselves most naturally to a processing account in which one inference is available prior to the other. 
are various proposals in the literature on how to generate it. Some argue that the non-conditional inference is also a presupposition (Schlenker 2011b,a; Singh 2007, 2008), while others argue that it is an inference generated based upon the conditional presupposition $\phi \rightarrow p$ (Pérez-Carballo 2009; von Fintel 2008; van Rooij 2007). The details of these proposals are not essential in the present context, but it should be noted that it is commonly assumed that whether the inference that $p$ obtains or not is determined by pragmatic factors.

\subsection{Conditional Entailment Theory}

Under the second theory, which we call the conditional entailment theory, conditional inferences are derived as mere entailments rather than as presuppositions (Gazdar 1979; Geurts 1999; van der Sandt 1992). One way to formalize this idea is to model presupposition resolution as integration of the presupposition into the discourse structure. It is assumed that the representation of a conditional sentence if $\phi$, then $\psi_{p}$ has at least two places where the presupposition $p$ can be integrated. One is the global position, in which case the resulting discourse ends up meaning $p$ and if $\phi$, then $\psi$, which captures the non-conditional inference. The other possibility is resolution in the local position within the consequent, in which case the resulting discourse means If $\phi$, then $p$ and $\psi$. This entails the conditional inference $\phi \rightarrow p$.

This theory assumes that global resolution is generally preferred, and local resolution becomes possible only in certain contexts. More specifically, Geurts (1999) suggests that local resolution becomes possible in the presence of a 'bridging' inference that if $\phi$, usually $p$ (see also Krahmer \& van Deemter 1998; Piwek \& Krahmer 2000). For an illustration, let us consider the examples (2) and (3) again. For (2), no bridging inference is readily available, and since the global option is preferred by assumption, the non-conditional inference that Paul has a Bible obtains. On the other hand for (3), a bridging inference that devout Catholics usually have a Bible makes the local reading plausible.

\subsection{Experimental Goals}

In this paper, we report on two experiments conducted on Amazon Mechanical Turk (MTurk). ${ }^{3}$ The main purpose of the experiments is to test the hypothesis that the conditional inference $\phi \rightarrow p$ is more likely to arise when the presupposition $p$ in the consequent is dependent on the antecedent $\phi$. This hypothesis is in principle compatible with both theories introduced above, as we will show in detail in Section 5. However we will also claim that the difference between the two experiments favors the conditional presupposition theory over the conditional entailment theory.

3 www.mturk.com/mturk/welcome 
Romoli, Sudo and Snedeker

\section{Experiment 1}

\subsection{Design}

Experiment 1 employs a picture matching task where participants read sentences and are asked to pick a picture that best matches what the sentences say. Participants were randomly assigned to either the Dependent condition or to the Independent condition. The critical items in the Dependent condition involve a conditional sentence of the form if $\phi$, then $\psi_{p}$ with a presupposition $p$ that is dependent on the antecedent $\phi$, while those in the Independent condition involve conditional sentences with a presupposition that is not dependent on the antecedent.

The conditional sentences for the two conditions were constructed based on an independent norming study that was also conducted on MTurk. In the norming study, participants were given a statement about a monster and were asked whether that statement made it more or less likely that the monster possessed a particular property (e.g. Googlemorph is flying. Does that make it more likely that he has wings?). The responses were given on a 5-point Likert scale, where 1 was "much less likely" and 5 was "much more likely". The eight items with the highest scores are used to construct the critical items of the Dependent condition, and the eight items whose scores were the closest to the middle point 2.5 were used to construct the critical items in the Independent condition.

Participants in each of the two conditions saw sixteen trials in total: four critical trials, four control trials and eight filler trials. The target and control trials involve conditional sentences with a possessive noun phrase. They are followed by a confirmation of the antecedent (control trials) or a denial of the antecedent (critical trials). Trial type (critical vs control) was counterbalanced across lists so that each item appeared in both forms across participants but each subject saw a given item in only one form. The sentences of a critical/control item from each condition are given in (5) and (6).

(5) Dependent

If Googlemorph is flying, then his wings are big and strong.

a. And Googlemorph is flying.

(Control)

b. But Googlemorph is not flying.

(Critical)

(6) Independent

If Googlemorph is drinking orange juice, then his wings are big and strong.

a. And Googlemorph is drinking orange juice.

(Control)

b. But Googlemorph is not drinking orange juice.

(Critical)

Each item has four pictures. In the control and critical items, the four pictures depict the following four monsters: (A) a monster (TTT) that satisfies all of the 


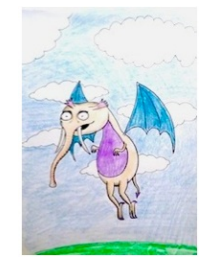

(A) : TTT

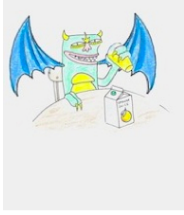

(A) : TTT

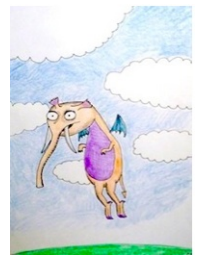

(B): TTF

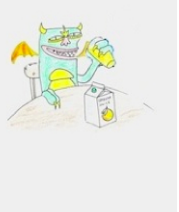

(B): TTF

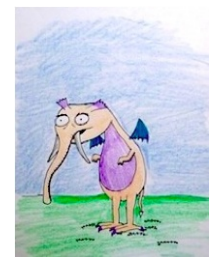

(C): FTF

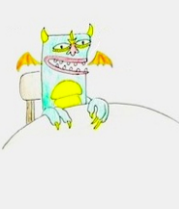

(C): FTF

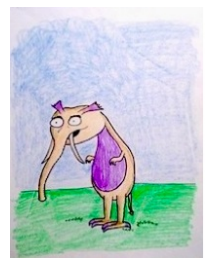

(D): FF-

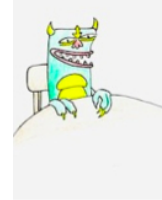

(D): FF-

Figure 1 The pictures used for (5) (top) and (6) (bottom) in Experiment 1. The letters represent the truth $(\mathrm{T})$ or falsity $(\mathrm{F})$ of the antecedent, the nonconditional presupposition and the consequent (e.g. TTT, Antecedent $=$ true, Presupposition $=$ true, Consequent $=$ true $)$.

antecedent, the non-conditional presupposition and the consequent; (B) a monster (TTF) that satisfies the antecedent and the non-conditional presupposition but not the consequent; (C) a monster (FTF) that does not satisfy the antecedent, satisfies the non-conditional presupposition and does not satisfy the consequent; and (D) a monster (FF-) that does not satisfy the antecedent or presupposition (and as a result the consequent is undefined). The pictures for the trials in (5) and (6) are as in Fig. 1.

In addition, participants saw eight filler items where the conditional sentences do not contain presuppositions in the consequent. In four of them, the conditional sentence is followed by a confirmation of the antecedent (7a) and in the other four, by a denial of it (7b).

\section{(7) Filler items}

a. If Googlemorph has red hair, he has yellow nails. And Googlemorph has red hair.

b. If Googlemorph has red hair, he has yellow nails. But Googlemorph doesn't have red hair.

In the filler trials, exactly one of the four pictures was compatible with the sentences and thus there was a single "right" answer. The control and filler items were intended to evaluate whether participants were attending and understood the directions. 

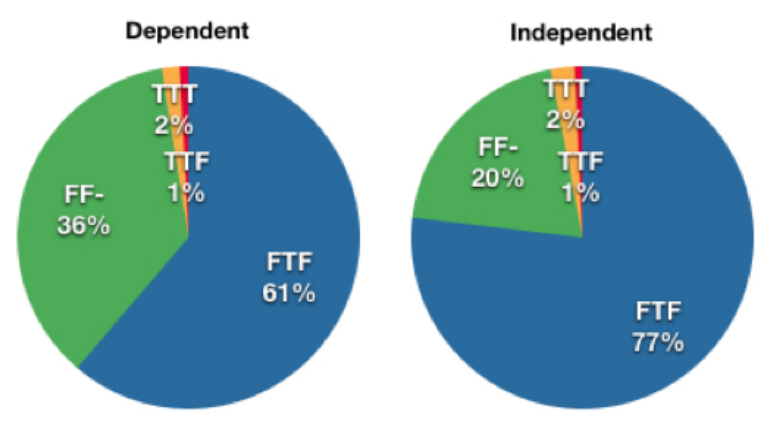

Figure 2 By-subject mean proportions of the answers for the critical items in Experiment 1.

The predictions are as follows. In the critical trials, the antecedent $\phi$ of the conditional if $\phi$, then $\psi_{p}$ is denied by the second sentence not- $\phi$, which makes the pictures TTT and TTF incompatible with the sentences. Crucially, if the conditional sentence has a non-conditional inference $p$, the picture FTF is the only compatible choice, while if it has a conditional inference $\phi \rightarrow p$, both the picture FTF and the picture FF- are compatible with the sentences. Under the hypothesis that conditional inferences are more likely when the presupposition of the consequent is dependent on the antecedent, the picture FF- is predicted to be chosen more often in the Dependent condition than in the Independent condition.

\subsection{Results and Discussion}

The data from 128 native speakers of English (61 participants in Dependent; 67 in Independent) are summarized in Fig. 2. Fifteen additional participants were tested but excluded from the analysis because they were non-native speakers of English (5) or their accuracy rate for the control and filler items is less than $80 \%$ (10). They were all paid $\$ 0.15$ for their participation.

As predicted, FF- was chosen significantly more often in the Dependent condition than in the Independent condition (Mann-Whitney $\mathrm{U}: U=2647.5, Z=3.22, p<$ .01). Moreover, in both conditions, FTF was chosen more often than FF- (Wilcoxon Signed Rank: Dependent: $W=300.5, Z=-215, p<.001$; Independent: $W=$ $70, Z=-6.24, p<.001)$.

These results suggest that participants are more likely to make conditional inferences when the presupposition is dependent on the antecedent, confirming the hypothesis. The preference for FTF might seem to suggest that the non-conditional inference was preferred in both conditions. This conclusion rests on the assumption 
that participants who arrived at a conditional inference would consistently select FF-. But the conditional inference is in principle compatible with both FF- and FTF. Therefore, while it is certain that a selection of FF- is due to the conditional inference, there is no direct way to know whether FTF is due to a conditional inference or non-conditional. In the next section, we present a modification of Experiment 1 that is designed to overcome this limitation, and in the subsequent section, we return to this feature of Experiment 1.

\section{Experiment 2}

\subsection{Design}

Experiment 2 is identical to Experiment 1 except that it employs the 'covered box' task of Huang, Spelke \& Snedeker (to appear). The covered box task is a variant of picture selection task where one of the pictures is covered and cannot be seen. In our experiment, participants were asked to select the covered picture only if none of the visible ones matched the sentences. ${ }^{4}$ In the critical trials of Experiment 2, FTF is covered. Given that the non-conditional inference is only compatible with this picture, while the conditional inference is compatible with both FTF and FF-, we expect that whenever the non-conditional inference arises, the covered picture will be selected, and furthermore that whenever the conditional inference arises, FF- will be selected.

Just as in Experiment 1, participants received both control items (antecedent confirmed) and critical items (antecedent denied) and this factor was counterbalanced in the same way as in Experiment 1. In control and filler trials the correct choice was visible for half of the trials and covered for the other half. The pictures for the trials in (5) and (6) for Experiment 2 are as in Fig. 3.

\subsection{Results and Discussion}

The data from 121 native speakers of English (59 in Dependent; 62 in Independent), none of whom participated in Experiment 1, are analyzed. 26 additional participants were tested but not included in the analysis because they were not native speakers of English (8) or their accuracy rate for the control and filler items was less than $80 \%$ (18). Participants were all paid $\$ 0.15$ for their participation. The results are summarized in Fig. 4, where CB stands for the covered box.

As in Experiment 1, we observe that FF- was chosen significantly more often in the Dependent condition than in the Independent condition (Mann-Whitney $\mathrm{U}: U=$

4 For other applications of the covered box task see Pearson, Khan \& Snedeker 2010 and Kotek, Sudo, Howard \& Hackl 2011. 

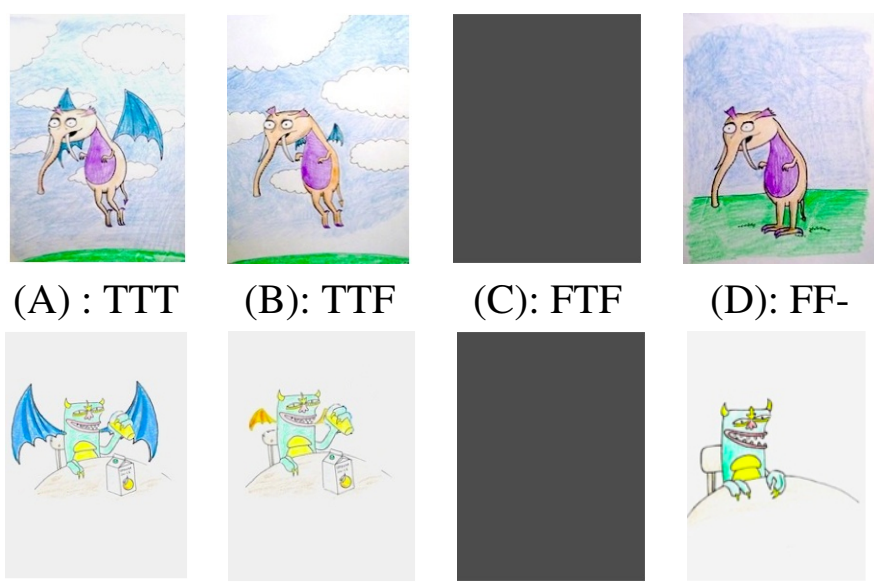

(B): TTF

(C): FTF

(D): FF-

(A) : TTT
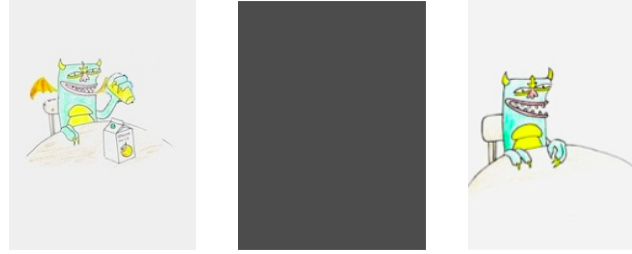

(B): TTF

(C): FTF

(D): FF-

Figure 3 The pictures used for (5) (top) and (6) (bottom) in Experiment 2. The letters represent the truth $(\mathrm{T})$ or falsity $(\mathrm{F})$ of the antecedent, the nonconditional presupposition and the consequent (e.g. TTT, Antecedent $=$ true, Presupposition $=$ true, Consequent $=$ true ) .

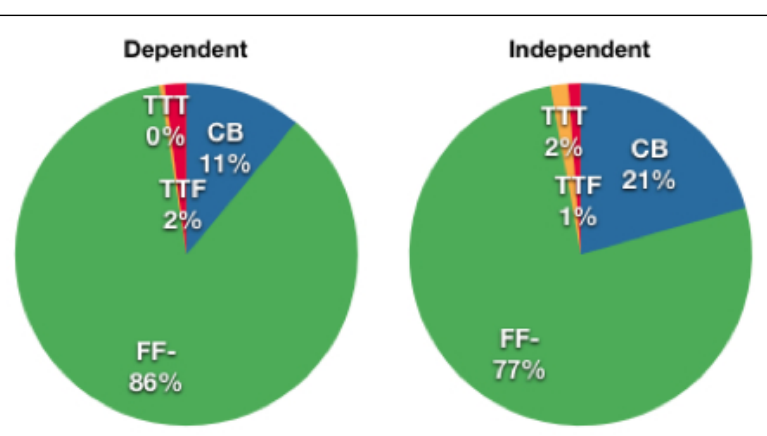

Figure 4 By-subject mean proportions of the answers to the critical items in Experiment 2. 
$2219, Z=2.26, p<.05)$, which replicates the results of Experiment 1. In addition, we also observe that FF- was selected more often than the covered box in both conditions (Wilcoxon Signed Rank: Dependent: $W=1583.5, Z=6.72, p<.001$; Independent: $W=1222.5, Z=5.98, p<.001)$. Critically, participants selected the covered box consistently in the control and filler trials where it was the only right answer (Dependent: $M=86.1 \%$; Independent: $M=83.9 \%$ ).

The fact that FF- was chosen more often in the Dependent condition in both Experiment 1 and Experiment 2 supports the hypothesis that dependency is a factor in the selection of the inference. Furthermore, it is also observed here that FF- is preferred to the covered box not only in the Dependent condition but also in the Independent condition. Recall that given the instructions that an overt picture should be chosen whenever possible, FF- is only compatible with the conditional inference. Thus it is suggested that the conditional inference is available in both conditions. In the following section, we consider the results of the two experiments in connection to the Generation Problem.

\section{General Discussion}

The results of the two experiments reported in the previous sections support the hypothesis that for conditional sentences of the form if $\phi$, then $\psi_{p}$, the dependency of the presupposition $p$ on the antecedent $\phi$ affects the choice of the inference. More specifically, a higher likelihood of $p$ given $\phi$, which in our case was measured according to naive intuitions in the norming study, leads to an increased chance of obtaining a conditional inference $\phi \rightarrow p$. To the best of our knowledge, this is the first experimental confirmation of the dependency hypothesis.

The effect of dependency itself is compatible with both approaches to the Generation Problem that we introduced in Section 2. The conditional presupposition theory would account for it with the selection mechanism that is sensitive to dependency. The conditional entailment theory, on the other hand, would account for it by assuming that the intuitive dependency between $p$ and $\phi$ makes more readily available the bridging inference that if $\phi$, then usually $p$.

Nevertheless, the present results place some constraints on solutions to the Generation Problem. Specifically, they suggest that conditional inferences are robustly available even when there is no dependence between the antecedent and the presupposition. To see this more clearly we must return to the results of Experiment 1 and consider the relation between the participant's interpretation of the sentences and the picture that they select.

Recall that in Experiment 1 the conditional presupposition is compatible with two pictures, namely FF- and FTF. That is, a conditional inference $\phi \rightarrow p$ is compatible 


\begin{tabular}{lll}
\hline & Dependent & Independent \\
\hline FF- & $35.6 \%$ & $19.5 \%$ \\
FTF & $61.9 \%$ & $77.7 \%$ \\
\hline
\end{tabular}

Table 1 Percentages of FF- versus FTF choices in Experiment 1.

\begin{tabular}{lll}
\hline & Dependent & Independent \\
\hline Conditional inference & $71.2 \%$ & $39 \%$ \\
Non-conditional inference & $26.3 \%$ & $58.2 \%$ \\
\hline
\end{tabular}

Table 2 Estimated percentages of conditionals and non-conditional inferences in Experiment 1 given assumption that participants who calculated conditional inferences were at chance in critical trials between FF- and FTF.

with not- $\phi$ but nonetheless $p$, which is the situation that FTF describes. ${ }^{5}$ Thus, while we know that the FF- choices are all due to participants who computed the conditional inference, when a participant chooses FTF we cannot be sure which inference they made. Thus the only way to estimate the proportion of conditional inferences is to make an additional assumption about what participants will do when they compute conditional inferences. The simplest assumption is that these participants will see the two possible pictures (FF- and FTF) as equally good depictions of the monster and thus will select one at random, choosing each one equally often. Let us now reconsider the results of Experiment 1, which are summarized in Table 1, in light of this assumption.

We know that in all trials in which the participant selected FF-, the conditional inference was selected, since the non-conditional inference is not compatible with this choice. Furthermore, if we accept the assumption above, then we know that an equal number of participants who made the conditional inference selected FTF since this inference provides no reasons for preferring FF- over FTF. This allows us to calculate estimates of the conditional inference $(2 * \mathrm{FF}$-) and the non-conditional inference (FTF-FF-). The estimated proportions of the conditional and non-conditional inferences are given in Table 2.

These estimates are important for two reasons. First, they suggest that the conditional inference was robustly available in Experiment 1 where the visible

5 In general a conditional sentence if $\phi$ then $\psi$ gives rise to the inference that if not- $\phi$ then not- $\psi$ (Geis \& Zwicky 1971 and much subsequent work), which is known as 'conditional perfection'. There is a question here as to whether the conditional inference $\phi \rightarrow p$ can further be interpreted to suggest $\neg \phi \rightarrow \neg p$. We leave this complication aside for now. 
alternatives might seem to favor the non-conditional inference. Participants in the Dependent condition preferred the conditional inference and even those in the Independent condition appear to have calculated it nearly half the time. Second, these estimates allow us to directly compare Experiment 1 and Experiment 2 and to examine the effects of the dependency manipulation in these two contexts. Curiously the effect of dependency seems to be greater in Experiment 1 than in Experiment 2. That is, the estimated percentage of the conditional inference in Experiment 1 is $71.2 \%$ in the Dependent condition and $39.0 \%$ in the Independent condition. For Experiment 2, as the two pictures directly correspond to the two inferences, the percentage of the conditional inference is $86 \%$ (Dependent) vs. $77 \%$ (Independent). We claim immediately below that under the simplest assumptions, this data is more straightforwardly explained by the conditional presupposition theory than by the conditional entailment theory.

To link the difference between the two experiments to the theories of presupposition projection, we must make some assumptions about how presuppositions are processed during language comprehension and how they guide performance during the task. In the case of the conditional presupposition theory it is most natural to assume that the conditional inference, which is available for all conditional sentences, is generated automatically upon reading the sentence. In contrast, the non-conditional inference only becomes available in contexts in which the antecedent and the presupposition are independent. In Experiment 1 both FF- and FTF are visible and we conjecture here that this makes salient the choice between the two inferences, and thus participants must go through the selection process. In Experiment 2, on the other hand, FF- is the only visible option compatible with the conditional presupposition, and hence the selection mechanism need not even be considered. Given that dependency is assumed to play a role only in the selection mechanism, the larger effect of dependency in Experiment 1 is accounted for. ${ }^{6}$

How does the conditional entailment theory explain the larger effect of dependency? Recall that the non-conditional inference is assumed to be the preferred option, unless a bridging inference is available. This is reflected in the results of Experiment 1. In Experiment 2, however, the visible picture was not compatible with the non-conditional inference, but the conditional entailment theory would have to say that participants generated a bridging inference rather than going for the covered box. Crucially this is also the case for the Independent condition, where the bridging inference is unnatural (e.g. monsters drinking orange juice usually have wings). In order for this to be the case, task constraints must be able to override the preference

6 Notice that the conditional presupposition theory augmented with the additional assumptions makes other testable predictions about the online processing. In particular we expect that the reaction times in Experiment 2 should be faster than in Experiment 1 and also in Experiment 2 we expect slower reaction times for the covered box than for visible pictures. We leave this for future research. 
for global resolution. However previous experiments that employed the covered box method (Huang et al. to appear; Pearson et al. 2010) suggest that participants did not hesitate to choose the covered box instead of canceling the optional, dispreferred inference inconsistent with the visible picture(s).

To sum up, the conditional presupposition theory more straightforwardly explains the difference between the two experiments, precisely because it postulates conditional presuppositions.

\section{Conclusions and Further Directions}

We have presented two picture selection experiments whose results support the hypothesis that the dependency between the antecedent and the presupposition plays a role in the selection of the inference, as previously suggested in the literature. This constitutes a partial answer to the Selection Problem, but questions remain about whether other factors play a role in selection and what notion of dependency underlies the intuitions of the participants of the norming study. ${ }^{7}$ Further research using these methods could address these questions by comparing different manipulations of the relation between the antecedent and presupposition.

As we noted above, these data also place some weak constraints on the Generation Problem. Specifically, two aspects of our data (the robust occurrence of the non-conditional inference and the difference in the dependency effect between Experiment 1 and Experiment 2) are more naturally accounted for under the conditional presupposition theory. These arguments, however, relied heavily upon auxiliary assumptions about language processing and how it is altered by the task itself. Thus our data does not conclusively rule out the conditional entailment theory.

In this final section, we sketch some possible further directions. In particular we discuss a previous experiment on the Generation Problem (Chemla \& Schlenker 2011), note one limitation of that study, and suggest an alternative way of investigating the Generation Problem.

As we have seen in Section 2, the conditional presupposition theory and the conditional entailment theory both account for conditional inferences but do so in different ways. For the conditional presupposition theory, conditional inferences are the presuppositions of conditional sentences, while for the conditional entailment theory, they are entailments resulting from local resolution of the presupposition. In order to tease apart the predictions of these theories, Chemla \& Schlenker (2011) used the additive presupposition trigger aussi (too in French), which is argued to resist local accommodation unlike possessive NPs. The following examples demonstrate the asymmetry between too and possessive NPs with respect to the

7 Thanks to Dan Lassiter (p.c.) for discussion on this point. 
Presupposition Projection in Conditional Sentences

availability of local accommodation.

a. I have talked to Ann. It's impossible that Bill will come in her car. Ann only has a motorbike.

b. I have talked to Ann. \#It's impossible that Bill will come too. Ann is abroad.

Specifically (8a) has a reading where the presupposition of the possessive NP that Ann owns a car is interpreted under the scope of impossible, and as a consequence the second sentence does not imply that Ann has a car. On the other hand, $(8 b)$ necessarily suggests that the presupposition triggered by too, i.e. that Ann will come, is true, which conflicts with the continuation Ann is abroad. This contrast is attributed to the property of too that its presupposition cannot be easily accommodated under the scope of an operator like impossible.

The logic behind Chemla \& Schlenker 2011 builds upon this property of too. Since local accommodation is unavailable for too, conditional sentences of the form if $\phi$, then $\psi$ with too occurring in $\psi$ requires the global resolution of the presupposition. As a consequence, the conditional entailment theory only predicts a non-conditional inference, but under the conditional presupposition theory the conditional presupposition is still available.

Chemla \& Schlenker (2011) use a task in which participants are shown conditional sentences like (9), and asked to judge whether the conditional inference (9a) and the non-conditional inference (9b) are robust inferences of (9) on a continuous scale ranging from no to yes. ${ }^{8}$ The English translations of one of their test sentences are reproduced below from Chemla \& Schlenker 2011.

(9) If Ann decides to study abroad, her brother will also make a reasonable decision.

a. Studying abroad would be reasonable for Anne.

b. Ann will make a reasonable decision.

They observe that the conditional inference is rated significantly higher than the nonconditional inference $(M=87 \%$ Yes vs. $M=58 \%$ Yes, $F(1,17)=32, p<.001)$, and they conclude that the results favor the conditional presupposition theory.

We would like to point out here that while these experimental results suggest that a version of the conditional entailment theory that relies on local accommodation is problematic, they do not provide conclusive evidence against all versions of the conditional entailment theory. That is, some versions of the conditional entailment theory use other mechanisms than local accommodation to derive the conditional

8 The experiment was conducted in French, but we only present the English translations for readability's sake. 
presupposition, which continue to be available for presuppositions triggered by too. In particular, Geurts (1999) suggests that for a conditional sentence a bridging inference can modify the discourse structure so that local resolution of the presupposition does not require the mechanism of local accommodation. More specifically, under Geurts's theory, local resolution and local accommodation of a presupposition are strictly distinguished. The former is only possible if the discourse structure already contains a resolution site with certain appropriate properties, while the latter is understood as a process of resolving the presupposition in a resolution site without these properties. Crucially, Geurts suggests that in the relevant cases the bridging inference creates a discourse structure with the properties necessary to locally resolve the presupposition. Therefore Chemla \& Schlenker's (2011) assumption that the conditional entailment theory does not predict conditional inferences for (9) is not necessarily valid, assuming that the bridging inference is available that studying abroad would (likely to) be a reasonable decision to Ann. Therefore the conditional entailment theory so understood is compatible with their experimental results.

As Chemla \& Schlenker (2011) themselves suggest, another way to investigate the question as to whether the conditional inference is a presupposition or a mere entailment is to check whether it projects as a presupposition. For example, when put in the antecedent of a conditional, the conditional inference is expected to project out only if it is a presupposition. On the other hand, the conditional entailment theory has no means to generate the conditional inference for the entire sentence. Notice importantly that this logic does not hinge on the assumption that the conditional entailment theory generates the conditional inference using local accommodation, unlike Chemla \& Schlenker 2011. We believe that the experimental task established in the present paper can be deployed in testing the predictions of the theories. We leave this for future research.

\section{References}

Beaver, David. 2001. Presupposition and Assertion in Dynamic Semantics. Stanford: CSLI Publications.

Beaver, David \& Bart Geurts. 2011. Presupposition. In Edward N. Zalta (ed.), The Stanford Encyclopedia of Philosophy, Summer 2011 edn.

Chemla, Emmanuel \& Philippe Schlenker. 2011. The reality of conditional presuppositions. Ms., LSCP, IJN and NYU.

Chierchia, Gennaro \& Sally McConnell-Ginet. 2000. Meaning and Grammar: An Introduction to Semantics. Cambridge, MA: MIT Press 2nd edn.

Gazdar, Gerald. 1979. Pragmatics: Implicature, Presupposition, and Logical Form. New York: Academic Press. 
Presupposition Projection in Conditional Sentences

Geis, Michael \& Arnold Zwicky. 1971. On invited inferences. Linguistic Inquiry 2(4). 561-565.

George, Benjamin. 2008. Presupposition Repairs: a Static, Trivalent Approach to Predicting Projection. University of California Los Angeles MA thesis.

Geurts, Bart. 1999. Presuppostions and Pronouns. Amsterdam: Elsevier.

Heim, Irene. 1983. On the projection problem for presuppositions. In Proceedings of West Coast Conference on Fornal Linguistics (WCCFL) 2, 114-125.

Heim, Irene. 1992. Presupposition projection and the semantics of attitude verbs. Journal of Semantics 9(3). 183-221. doi:10.1093/jos/9.3.183.

Huang, Yi Ting, Elisabeth Spelke \& Jesse Snedeker. to appear. What exactly do numbers mean? Language, Learning and Development .

Kadmon, Nirit. 2001. Formal Pragmatics. Oxford: Blackwell.

Karttunen, Lauri. 1974. Presupposition and linguistic context. Theoretical Linguistics 1(1-3). 181-194. doi:10.1515/thli.1974.1.1-3.181, //1974.

Kotek, Hadas, Yasutada Sudo, Edwin Howard \& Martin Hackl. 2011. Most meanings are superlative. In Jeff Runner (ed.), Syntax and Semantics 37: Experiments at the Interface, 101-145. New York: Academic Press.

Krahmer, Emiel \& Kees van Deemter. 1998. On the interpretation of anaphoric noun phrases: Towards a full understanding of partial matches. Journal of Semantics 15(4). 355-392. doi:10.1093/jos/15.4.355.

Pearson, Hazel, Manizeh Khan \& Jesse Snedeker. 2010. Even more evidence for the emptiness of plurality: An experimental investigation of plural interpretation as a species of implicature. In Semantics and Linguistic Theory (SALT) 20, 489-508.

Pérez-Carballo, Alejandro. 2009. Toward a dissolution of the proviso problem. In Paul Egré \& Giorgio Magri (eds.), Proceedings of MIT-France Workshop on Scalar Implicatures and Presuppositions, 169-184. Cambridge, MA: MITWPL.

Piwek, Paul \& Emiel Krahmer. 2000. Presuppositions in context: Constructing bridges. In Pierre Bonzon, Marcos Cavalcanti \& Rolf Nossum (eds.), Formal Aspects of Context, Dordrecht: Kluwer.

Schlenker, Philippe. 2008. Be Articulate! A pragmatic theory of presupposition projection. Theoretical Linguistics 34(3). 157-212. doi:10.1515/THLI.2008.013, /December/2008.

Schlenker, Philippe. 2009. Local contexts. Semantics and Pragmatics 2. 1-78. doi:10.3765/sp.2.3.

Schlenker, Philippe. 2010. Local contexts and local meanings. Philosophical Studies 151(1). 115-142. doi:10.1007/s11098-010-9586-0.

Schlenker, Philippe. 2011a. DRT with local contexts. Natural Language Semantics doi:10.1007/s11050-011-9069-7.

Schlenker, Philippe. 2011b. The proviso problem: a note. Natural Language Semantics doi:10.1007/s11050-011-9072-z. 
Singh, Raj. 2007. Formal alternatives as a solution to the proviso problem. In Semantics and Linguistic Theory (SALT), vol. 17, 264-281.

Singh, Raj. 2008. Modularity and Locality in Interpretation: MIT dissertation. van der Sandt, Rob. 1992. Presupposition projection as anaphora resolution. Journal of Semantics 9(4). 333-377. doi:10.1093/jos/9.4.333.

van Rooij, Robert. 2007. Strengthening conditional presuppositions. Journal of Semanics 24(3). 289-304. doi:10.1093/jos/ffm007.

von Fintel, Kai. 2008. What is accommodation, again? Philosophical Perspectives 22(1). 137-170. doi:10.1111/j.1520-8583.2008.00144.x.

Jacopo Romoli

309, Boylston Hall

Department of Linguistics

Harvard University

Cambridge, MA

jromoli@fas.harvard.edu

Jesse Snedeker

Department of Psychology

Harvard University

snedeker@wjh.harvard.edu
Yasutada Sudo

Department of Linguistics and Philosophy Massachusetts Institute of Technology

77 Massachusetts Avenue, 32-D808

Cambridge, MA

ysudo@mit.edu 